\title{
EpiMethylTag: simultaneous detection of ATAC-seq or ChIP-seq signals with DNA methylation
}

\author{
Priscillia Lhoumaud', Gunjan Sethia', Franco Izzo ${ }^{2,3}$, Theodore Sakellaropoulos ${ }^{1,4}$, Valentina Snetkova', \\ Simon Vidal ${ }^{5}$, Sana Badri ${ }^{1}$, Macintosh Cornwell', Dafne Campigli Di Giammartino ${ }^{6}$, Kyu-Tae Kim², ${ }^{2,}$ Effie Apostolou ${ }^{6}$, \\ Matthias Stadtfeld ${ }^{5}$, Dan Avi Landau ${ }^{2,3,7}$ and Jane Skok ${ }^{1,5^{*}}$ (D)
}

\begin{abstract}
Activation of regulatory elements is thought to be inversely correlated with DNA methylation levels. However, it is difficult to determine whether DNA methylation is compatible with chromatin accessibility or transcription factor (TF) binding if assays are performed separately. We developed a fast, low-input, low sequencing depth method, EpiMethylTag, that combines ATAC-seq or ChIP-seq (M-ATAC or M-ChIP) with bisulfite conversion, to simultaneously examine accessibility/TF binding and methylation on the same DNA. Here we demonstrate that EpiMethylTag can be used to study the functional interplay between chromatin accessibility and TF binding (CTCF and KLF4) at methylated sites.
\end{abstract}

Keywords: DNA methylation, Chromatin accessibility, ChIP, ATAC, CTCF, KLF4

\section{Introduction}

The role of DNA methylation (DNAme) in gene regulation has been widely described [1-4]. In general, methylation is thought to reduce accessibility and prohibit TF binding at enhancers and promoters [5, 6]. Nevertheless, TFs are also known to bind methylated DNA [2], but due to limitations in the techniques available for this kind of analysis, few genomewide studies have been performed. As a result, we still know very little about the DNA sequence and chromatin context of TF binding at methylated sites and its significance to gene regulation.

Several techniques have been developed to measure DNAme, some more comprehensive than others. Whole-genome bisulfite sequencing (WGBS) covers all genomic regions; however, to achieve sufficient sequencing, coverage is costly. The alternative, reduced representation bisulfite sequencing (RRBS),

\footnotetext{
* Correspondence: Jane.Skok@nyulangone.org

${ }^{1}$ New York University Langone Health, New York, NY, USA

${ }^{5}$ Skirball Institute of Biomolecular Medicine, Department of Cell Biology, Helen L. and Martin S. Kimmel Center for Biology and Medicine, Laura and Isaac Perlmutter Cancer Center, New York, NY, USA

Full list of author information is available at the end of the article
}

which requires less sequencing depth, preferentially captures CpG-dense sequences known as CpG islands that can potentially act as regulatory elements [7]. Nevertheless, both techniques require additional assays on different batches of cells to elucidate the interplay between DNAme, DNA accessibility, and TF binding, and this does not satisfactorily address the issue of compatibility. Current techniques that simultaneously analyze methylation together with TF binding or accessibility (NOME-seq [8], HT-SELEX [9], ChIP-bisulfite [10], BisChIP-seq [11], ChIP-BisSeq [12]) have drawbacks such as analysis of DNA rather than chromatin or the requirement of large amounts of input DNA or high sequencing costs.

To circumvent the high input and sequencing expenses associated with WGBS and existing ChIP combined with bisulfite conversion protocols [1012], we developed "EpiMethylTag." This technique combines ATAC-seq or ChIPmentation [13, 14] with bisulfite conversion (M-ATAC or M-ChIP, respectively) to specifically determine the methylation status of accessible or TF-bound regions in a chromatin context. EpiMethylTag is based on an approach that was originally developed for tagmentation-based

(c) The Author(s). 2019 Open Access This article is distributed under the terms of the Creative Commons Attribution 4.0 International License (http://creativecommons.org/licenses/by/4.0/), which permits unrestricted use, distribution, and reproduction in any medium, provided you give appropriate credit to the original author(s) and the source, provide a link to the Creative Commons license, and indicate if changes were made. The Creative Commons Public Domain Dedication waiver (http://creativecommons.org/publicdomain/zero/1.0/) applies to the data made available in this article, unless otherwise stated. 
WGBS [15, 16]. It involves use of the Tn5 transposase, loaded with adapters harboring cytosine methylation (Additional file 2: Table S1).

For M-ATAC or M-ChIP, tagmentation occurs respectively on nuclear lysates as per the conventional ATAC-seq protocol [13], or during chromatin immunoprecipitation as per the ChIPmentation protocol [14]. Following DNA purification, the sample is bisulfite converted and PCR amplified for downstream sequencing (Fig. 1a). As shown in Fig. 1a, EpiMethylTag can determine whether DNAme and accessibility/ TF binding are mutually exclusive (scenario 1) or can coexist in certain locations (scenario 2). The protocol requires lower levels of immunoprecipitated DNA, requires less sequencing depth, is quicker than existing methods, and can be analyzed using a pipeline we developed that is publicly available online on Github (https://github.com/skoklab/EpiMethylTag).

a Schematics of the EpiMethylTag method

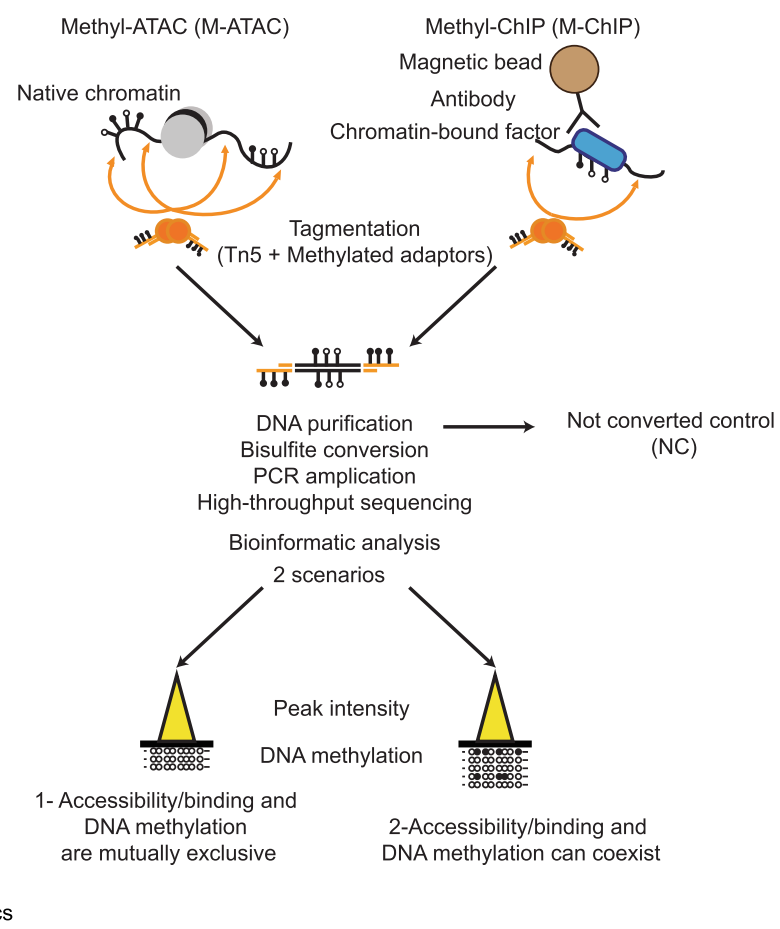

b Sequencing metrics

\begin{tabular}{|c|c|c|c|c|c|}
\hline & $\begin{array}{c}\text { raw reads } \\
\text { counts } \\
\text { (million) }\end{array}$ & Alignment $\%$ & $\begin{array}{c}\text { duplication } \\
\%\end{array}$ & peaks \# & $\begin{array}{c}\text { Fraction of } \\
\text { reads in } \\
\text { peaks (\%) }\end{array}$ \\
\hline Methyl-ATAC-rep3 & 25.70 & 62.61 & 54.30 & 31907 & 6.25 \\
\hline Methyl-ATAC-rep4 & 19.66 & 63.52 & 46.94 & 30413 & 6.26 \\
\hline ATAC_mESC-1 & 42.70 & 63.55 & 47.81 & 28717 & 4.22 \\
\hline ATAC_mESC-2 & 43.67 & 64.65 & 43.60 & 37537 & 5.25 \\
\hline ATAC_Tn5me-NC-1 & 32.35 & 67.00 & 64.70 & 31806 & 8.03 \\
\hline ATAC_Tn5me-NC-2 & 35.73 & 67.85 & 61.53 & 36710 & 9.09 \\
\hline CTCF Methyl-ChIP-rep1 & 25.91 & 66.72 & 71.74 & 33898 & 9.70 \\
\hline CTCF Methyl-ChIP-rep2 & 22.54 & 69.48 & 85.01 & 32195 & 11.91 \\
\hline CTCF ChIP-BisSeq (GSE39739) & 264.78 & 32.80 & 58.36 & 64460 & 10.49 \\
\hline CTCF ChIP-rep1 & 29.56 & 81.79 & 34.77 & 41731 & 8.18 \\
\hline CTCF ChIP-rep2 & 32.38 & 82.98 & 39.73 & 52327 & 14.19 \\
\hline CTCF Methyl-ChIP-NC-rep1 & 30.95 & 63.11 & 85.11 & 22576 & 7.85 \\
\hline CTCF Methyl-ChIP-NC-rep2 & 29.56 & 75.68 & 85.17 & 31409 & 12.81 \\
\hline WGBS & 257.12 & 74.98 & 8.27 & NA & NA \\
\hline
\end{tabular}

Fig. 1 EpiMethylTag is a reproducible method to test whether DNAme can coexist with TF binding (CTCF) or chromatin accessibility. a Schematic overview of the EpiMethyTag method showing two possible outcomes. b Sequencing metrics indicating the total number of reads in million, the alignment and duplication percentages, the number of peaks, and the fraction of reads in peaks (in percentage) for each sample as compared to public data (CTCF ChIP-BisSeq and WGBS) 


\section{Results}

EpiMethylTag is a reproducible method for testing the compatibility of DNAme with TF binding or chromatin accessibility

M-ATAC and CTCF M-ChIP were performed in duplicate on murine embryonic stem cells (mESC). As controls, we collected aliquots before bisulfite conversion, ATAC-seq, and CTCF ChIPmentation with Nextera transposase $[13,14]$. Sequencing metrics are shown in Fig. $1 \mathrm{~b}$ and Additional file 2: Table S2. The price is around 10 times lower than WGBS given that fewer reads are necessary. As shown in Fig. 2 a and b, genome coverage was highly reproducible between M-ATAC replicates and highly correlated with regular ATAC-seq and M-ATAC signal before bisulfite treatment. Thus, bisulfite treatment, or the use of a different transposase does not result in signal bias. High reproducibility was also seen for CTCF M-ChIP, and we observed consistency between our results and data generated by CTCF ChIP-BisSeq, a similar technique that was performed using $100 \mathrm{ng}$ of immunoprecipitated DNA (as opposed to less than $1 \mathrm{ng}$ using our method) and sequenced more deeply at a higher cost [12] (Fig. 2a, b, Additional file 2: Table S2). Of note, bisulfite conversion does not affect the number of peaks detected, the Jaccard index of peak overlap (Additional file 1: Figure S1ab), or the signal within peaks (Additional file 1: Figure S1c, Pearson correlations above 0.8 ), although it leads to shorter reads (Additional file 1: Figure S2). Of note, average methylation was higher at the edges of the peaks than at the midpoint (Additional file 1: Figure S3). Comparable DNA methylation levels were found in M-ATAC and CTCF M-ChIP replicates, Pearson correlation $=0.76$ and 0.84, respectively (Additional file 1: Figure S4a and S4b).

In order to get higher coverage for subsequent DNA methylation analysis, peaks were called from merged MATAC and M-ChIP replicates and we focused our analysis only at $\mathrm{CpGs}$ within those peak regions covered by at least five reads, as methylation outside of M-ATAC and M-ChIP peaks has low coverage and is less reliable. We observe positive correlations between DNA methylation from WGBS and M-ATAC (Fig. 2c, top panel, Pearson correlation $=0.69$ ) and between methylation levels in M-ChIP and WGBS (Fig. 2c, bottom panel, Pearson correlation $=0.74$ ). Similar results were observed with the previously published CTCF ChIP-BisSeq method [12] (GSE39739) (Pearson correlation $=0.83$, Additional file 1: Figure S4c) and when taking peaks that overlap between duplicates (Additional file 1: Figure S4d-e). In Fig. 2b, we highlight the Klf4 gene, which harbors a peak of chromatin accessibility in the promoter and CTCF binding in the intragenic region associated with low methylation from both EpiMethylTag and WGBS assays (left panel, and Additional file 2: Table S3). In contrast, the Pisd-ps1 intragenic region contains accessible chromatin that coexists with high levels of DNA methylation as detected by both M-ATAC and WGBS (Fig. 2b, middle panel). Of note, the methylation observed comes from a bedGraph file, output from Bismark (see "Methods" section for details), which does not filter for cytosines with low read coverage. Therefore, high methylation observed in CTCF M-ChIP may not be reliable as this region harbors a weak CTCF signal with a low read coverage (Additional file 2: Table S4). Interestingly, a proportion of MATAC peaks exhibited an intermediate-to-high average methylation level in deeply sequenced WGBS [17], but low methylation in M-ATAC (Fig. 2c, top panel, top left corner) as illustrated at the Slc5a8 locus (Fig. 2b, right panel, Additional file 2: Table S5). The peak highlighted within the Slc5a8 locus harbors an average methylation of $18.685 \%$ for M-ATAC and $85.041 \%$ for WGBS. These data suggest that as expected open regions are less methylated than closed regions within a population of cells, but that accessibility and methylation can coexist at a small subset of genomic locations, which are depleted for promoter regions and associated with low transcription (Additional file 1: Figure S4f-g). Importantly, M-ATAC is able to identify methylation levels within ATAC peaks, information that cannot be retrieved integrating data from separate WGBS and ATAC-seq experiments.

\section{M-ATAC reveals a complex interplay between accessible chromatin and DNA methylation}

For further analysis, we separated CpGs in M-ATAC peaks according to percentage of methylation (low 0 $20 \%$, intermediate $20-80 \%$, and high $>80 \%$ ) and read coverage (high $>50$ reads and low 5-50 reads) as follows: \#1: Low methylation/High coverage (22,932 CpGs); \#2: Low Methylation/Low coverage (1,348,931 CpGs); \#3: Intermediate methylation/Low coverage (39,321 CpGs); \#4: High methylation/Low coverage (1652 CpGs) (Fig. 3a). As expected, coverage and methylation from M-ATAC are anticorrelated, and we did not detect any CpGs with intermediate or high methylation with high ATAC coverage ( $>50$ reads). A similar pattern was observed while taking only CpGs present in peaks that overlap between M-ATAC replicates (Additional file 1: Figure S5a). Of note, this pattern was not detected in WGBS where a more stable coverage is observed independent of methylation levels resulting in only three groups (Additional file 1: Figure S5b) as opposed to the four groups seen with methyl-ATAC (Fig. 3a). CpGs in low methylation M-ATAC groups 1 and 2 were enriched at promoters, while $\mathrm{CpGs}$ in intermediate and high methylation M-ATAC groups 3 and 4 were enriched in intragenic and intergenic regions, as compared to the 
a

Pearson Correlation of Read Counts

M-ATAC

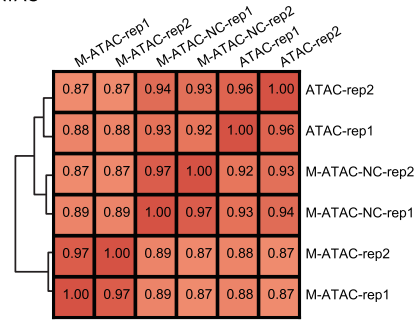

CTCF M-ChIP

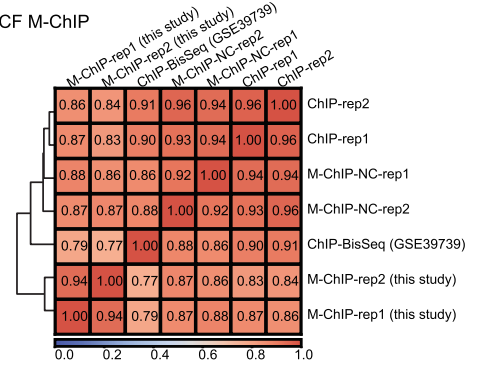

C Average methylation from EpiMethylTag compared to WGBS
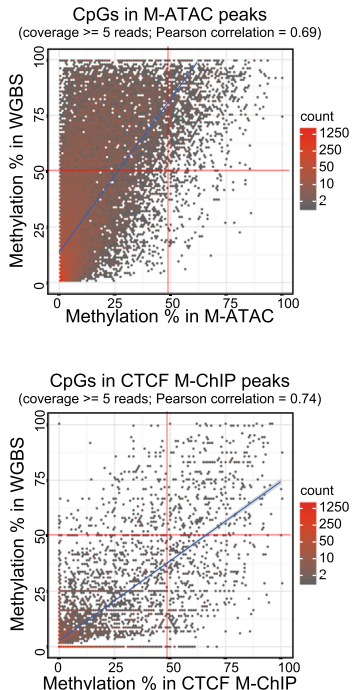

b

EpiMethylTag at KIf4 locus

EpiMethylTag at Pisd-ps 1 locus

EpiMethylTag at S/c5a8 locus

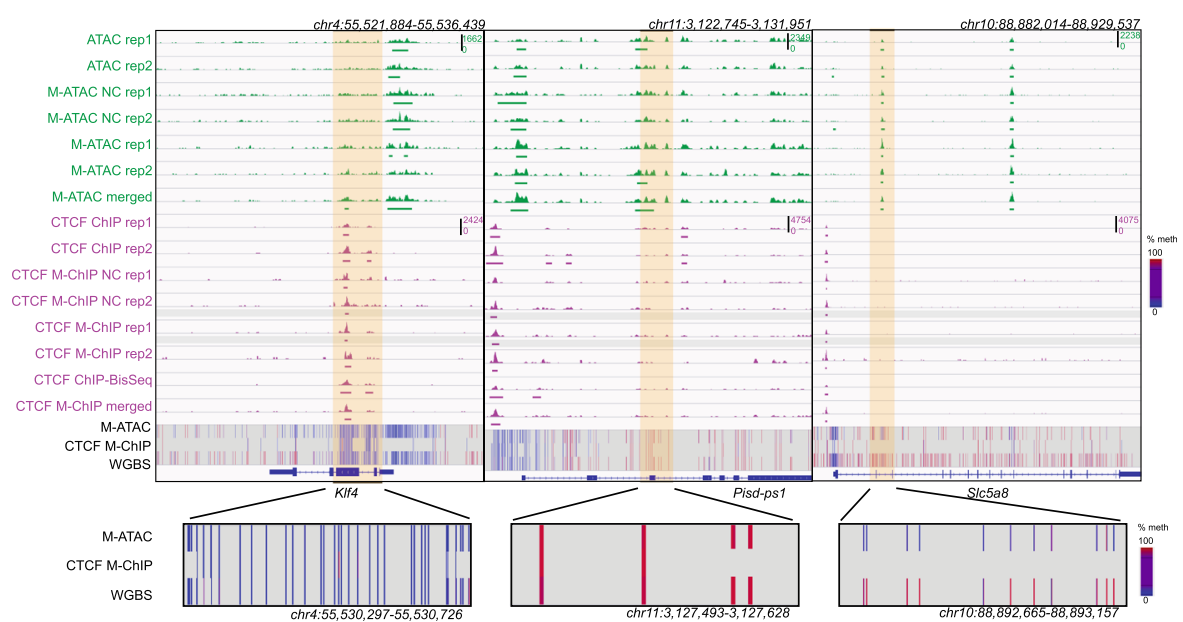

Fig. 2 EpiMethylTag is a reproducible method for testing whether DNAme can coexist with TF binding (CTCF) or chromatin accessibility genomewide. a Pearson correlation of read counts comparing M-ATAC with unconverted samples (NC) and regular ATAC-seq (top), and CTCF M-ChIP with unconverted samples, a sample from the Schubeler lab generated using ChIP-BisSeq [1] (GSE39739) and regular CTCF ChIP-seq (bottom). b Representative IGV screenshots of EpiMethylTag, at the KIf4 locus (left panel), the Pisd-ps1 locus (middle panel), and the S/c5a8 locus (right panel). ATAC and M-ATAC in green, CTCF in purple and DNA methylation from merged M-ATAC, merged CTCF M-ChIP and WGBS (methylation from 0\% in blue to $100 \%$ in red). A zoom-in of methylation at the highlighted region is shown at the bottom of each example. The Klf4 locus illustrates a region that has low methylation as detected by M-ATAC, CTCF M-ChIP, and WGBS. The Pisd-ps 1 locus illustrates a region that has high methylation as detected by M-ATAC, CTCF M-ChIP, and WGBS. The S/C5a8 locus illustrates a region that has low methylation as detected by MATAC and high methylation as detected by WGBS. c Density plots of methylation from EpiMethyltag compared with WGBS. Only CpGs inside peaks and with at least five reads were considered. Top: average methylation of CpGs per M-ATAC peak in M-ATAC versus WGBS (Pearson correlation $=0.69, p$ value $<2.2 \mathrm{e}-16$; bottom left corner: 27977 peaks, top left corner: 8408 peaks, top right corner: 1019 peaks, bottom right corner: 113 peaks). Bottom: average methylation per CTCF M-ChIP peak of CpGs in CTCF M-ChIP versus WGBS (Pearson correlation $=0.74, p$ value <2.2e-16; bottom left corner: 6549 peaks, top left corner: 198 peaks, top right corner: 304 peaks, bottom right corner: 310 peaks)

full set of M-ATAC peaks (Fig. 3b). The average methylation was more negatively correlated with transcriptional output for CpGs at promoters (Fig. 3c) than for intragenic CpGs (Additional file 1: Figure S5c). Heatmaps for M-ATAC read coverage intensity highlight the reproducibility of signal between individual replicates. Merged replicates were used for downstream analysis (Additional file 1: Figure S5d). Intriguingly, H3K4me1 showed a pronounced enrichment at CpGs with high levels of methylation (group 4) at promoter regions 
CpGs from M-ATAC separared into four groups according to read density and methylation

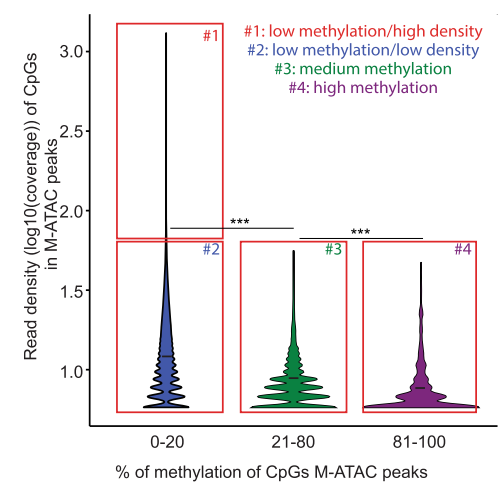

Expression level of genes associated C with the four groups of methylated CpGs at promoter locations.

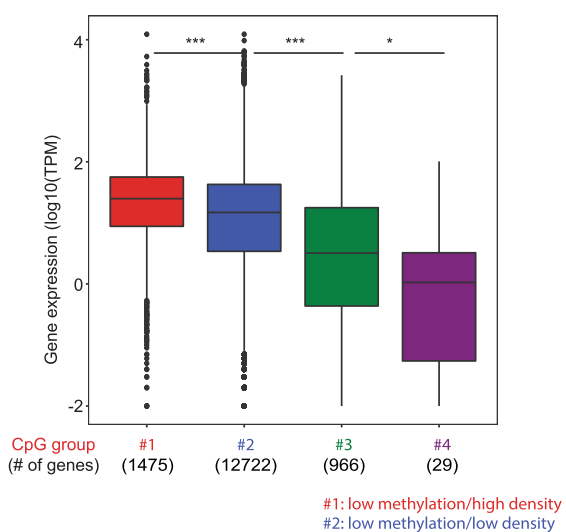

b

Genomic annotations of M-ATAC peaks associated

with the four groups of methylated $\mathrm{CpGs}$

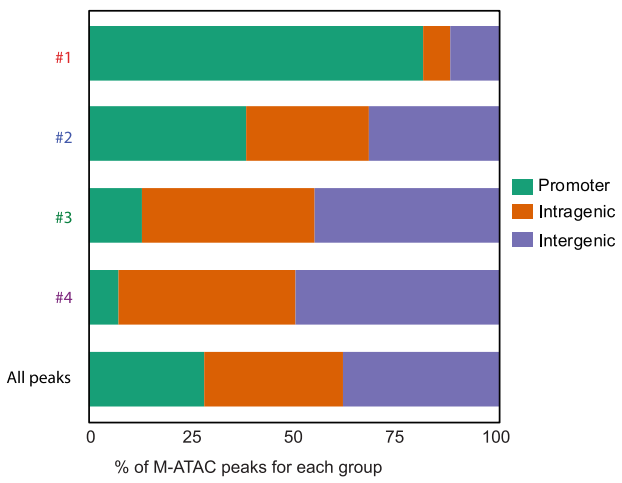

d

Profile of M-ATAC and Histone modifications in the four groups of methylated $\mathrm{CpGs}$
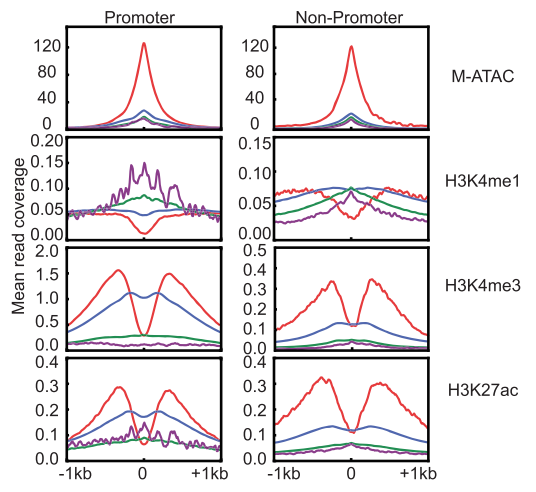

\#3: medium methylatio

Fig. 3 M-ATAC reveals a complex interplay between accessible chromatin and DNA methylation. a CpGs in M-ATAC peaks from merged replicates were divided into four groups according to methylation and coverage status: 1. Low Methylation $(<20 \%)+$ High coverage $(>50$ reads) (22,932 CpGs). 2. Low Methylation + Low coverage (5 to 50 reads) (1,348,931 CpGs). 3. Intermediate methylation (20-80) + Low coverage (5 to 50 reads) (39,321 CpGs). 4. High methylation (> 80\%) + Low coverage (5 to 50 reads) (1652 CpGs). *** $p<1$ e-300 between groups \#1 + 2 and group \#3, ${ }^{* * *} P=3.25 \mathrm{e}-109$ between groups \#3 and 4 (Wilcoxon text). b Genomic annotations for M-ATAC peaks corresponding to the 4 groups from Fig. 3a as well as the full list of M-ATAC peaks. Promoter: TSS $-3 \mathrm{~kb}$ and $+3 \mathrm{~kb}$; intragenic: introns, exons, 5'UTR, $3^{\prime} U T R$ and TTS, intergenic: distal from promoter $>1 \mathrm{~kb}$ and non-coding RNAs. c Expression level of genes associated with the four groups of methylated CpGs from in Fig. 3a, for the CpGs at promoters. ${ }^{* *} P=4.2 \mathrm{e}-33$ between groups $\# 1$ and $2,{ }^{* * *} P=2.8 \mathrm{e}-75$ between groups $\# 2$ and $3,{ }^{*} P=0.034$ between groups $\# 3$ and 4 (Wilcoxon test). d Average profile of M-ATAC, H3K4me1, H3K4me3, and H3K27ac signal associated with the four groups of methylated CpGs from Fig. $3 a$ at promoters versus non-promoters. Of note, the small number of promoters in group 4 gives an unsmooth pattern for marks such as H3K4me1 and H3K27ac

(Fig. 3d and Additional file 1: Figure S5e). In contrast, $\mathrm{H} 3 \mathrm{~K} 27 \mathrm{ac}$ and H3K4me3 were enriched at CpGs with low levels of methylation (groups 1 and 2), for both promoters and non-promoters.

\section{CTCF M-ChIP enables analysis of DNA methylation of distinct CpGs in the CTCF motif}

As a case study, CTCF M-ChIP was used to analyze the impact of DNAme on CTCF binding in M-ATAC peaks harboring a CTCF motif (Fig. 4a, top panel). M-ATAC groups 2 and 3 comprise the vast majority of $\mathrm{CpGs,}$ more CTCF peaks, motifs, and a proportionally higher number of CpGs within CTCF motifs (Additional file 1:
Figure S5f). However, the percentage of CpGs within CTCF motifs in each group is fairly constant: between 1.26 and $1.93 \%$ of CpGs). Of note, de novo CTCF motifs in CTCF ChIP-seq and Methyl-ChIP peaks were comparable to the MA0139.1 motif from the Jaspar database (Additional file 1: Figure S6a). CTCF occupancy has been inversely correlated with DNA methylation [18]. This finding is consistent with our analyses (Additional file 1: Figure S6b-d). Although CTCF peaks are associated with all levels of CpG methylation within CTCF motifs, as illustrated in Additional file 1: Figure S6e, the majority of CTCF peaks harbor reduced methylation (Additional file 1: Figure S6f). In the context of 


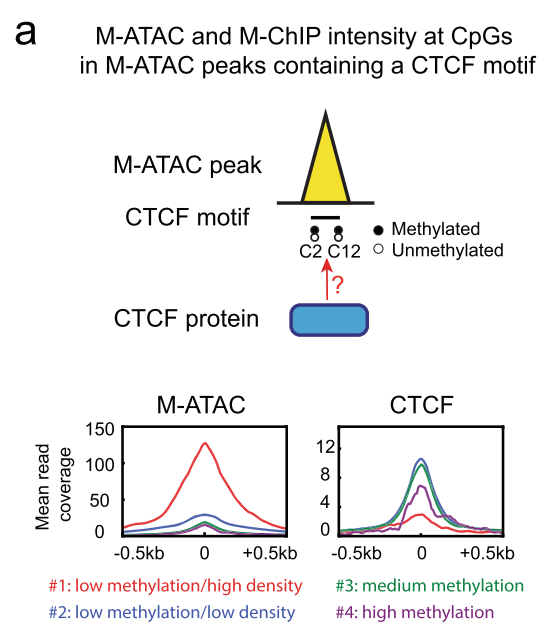
C Relationship between binding strength and
DNA methylation within the KLF4 M-ChIP peaks (coverage $>=5$ reads; Pearson correlation $=0.25$ )

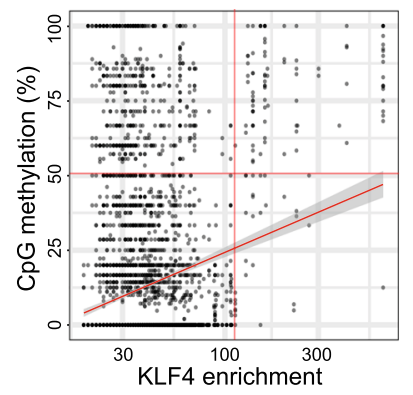
b Methylation $\%$ of CpGs in CTCF motif
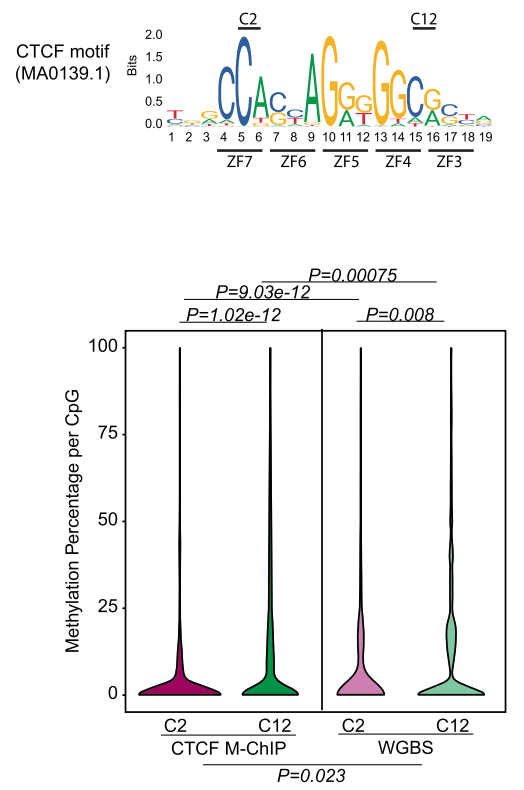

d

Intersection of KLF4 M-ChIP WT vs mutant

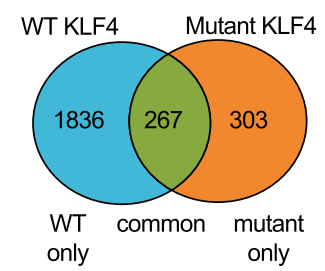

Pelative distribution of KLF4 motifs in WT only, mutant only and common KLF4 M-ChIP peaks

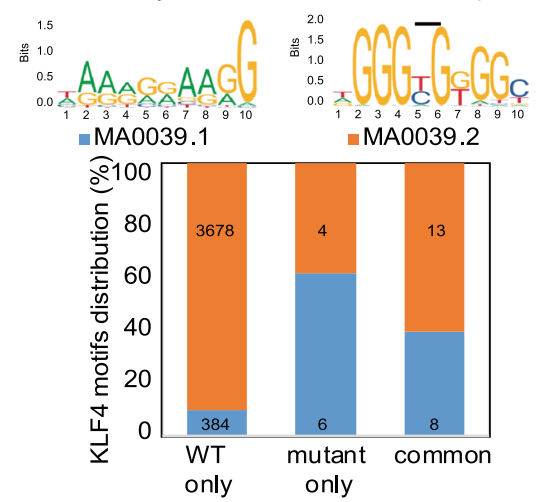

f Heatmaps of M-ATAC signal at WT only, Mutant only and common peaks

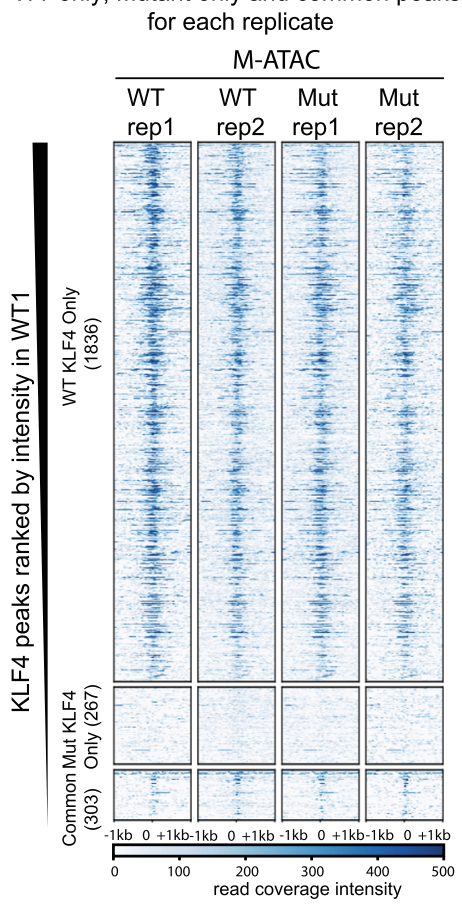

9 CpG methylation in KLF4 WT-only $\mathrm{M}-\mathrm{ChIP}$ peaks (coverage $>=5$ reads)

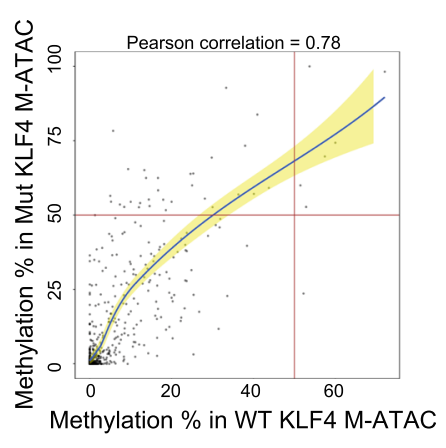

Fig. 4 M-ChIP enables analysis of DNA methylation binding by CTCF and KLF4. a Top: Schematic illustration representing an ATAC-seq peak with a CTCF motif and CTCF occupancy dependent on C2 and C12 methylation. Bottom: average profiles of M-ATAC (left) and CTCF M-ChIP (right) intensity at CpGs in a CTCF motif within M-ATAC peaks for the four groups of CpGs (group \#1: 288 CpGs, group \#2: 17133 CpGs, group \#3 CpGs: 758, group \#4: 25 CpGs). b top: CTCF motif from JASPAR database (MA0139.1). The 2 key CpG positions (C2 and C12) are indicated. Bottom: violin plots of methylation percentage from CTCF M-ChIP and WGBS, at C2 and C12 positions in the CTCF motif (MA0139.1). ***P=1.02e-12 for C2 CTCF M-ChIP versus C12 CTCF M-ChIP (Wilcoxon test), ${ }^{* *} P=0.008$ for C2 WGBS versus C12 WGBS (Wilcoxon test), *** $P=9 \mathrm{e}-12$ for C2 CTCF MChIP versus C2 WGBS (Wilcoxon test, paired), ${ }^{* *} P=0.00075$ for C12 CTCF M-ChIP versus C12 WGBS (Wilcoxon test, paired), ${ }^{*} P=0.023$ for CTCF MChIP versus WGBS (logistic regression model). c Scatter plot showing the relationship between binding strength and CpG methylation within the KLF4 M-ChIP peaks (Pearson correlation = 0.25; bottom left corner: 5138 CpGs, top left corner: 578 CpGs, top right corner: 104 CpGs, bottom right corner: 60 CpGs). d Venn diagram showing the overlap between WT and mutant KLF4 M-ChIP peaks. e Top: Illustration of KLF4 motifs from the Jaspar database (MA0039.1 and MA0039.2). The black bar represents the potential CpGs present in the MA0039.2 motif. Bottom: histogram showing the relative distribution of KLF4 motifs in WT, mutant and common KLF4 M-ChIP peaks using FIMO from the MEME suite. Absolute numbers of each motif are indicated. $\mathbf{f}$ Heatmap showing M-ATAC signal intensity at KLF4 M-ChIP peaks that are specific to WT (1836 peaks), mutant (267 peaks), or common between both conditions (303 peaks). g Average cytosine methylation from M-ATAC in WT versus mutant KLF4 expressing cells in WT specific KLF4 M-ChIP peaks (Pearson correlation $=0.78, p$ value $<2.2 \mathrm{e}-16$ ) 
CpGs in M-ATAC peaks, our data also demonstrates that the CTCF motif has an enriched CTCF intensity at CpGs with low and intermediate levels of methylation (groups 2 and 3) compared to CpGs with low and high levels of methylation (groups 1 and 4) (Fig. 4a, bottom panel). The highest binding is found in groups 2 and 3, compared to groups 1 and 4 that harbor reduced CTCF enrichment. Group 2 displays a wide range of accessibility (Additional file 1: Figure S5d-e), with the most open regions of group 2 resembling group 1, and the most closed regions of this group being similar to that of group 3. Interestingly, even though there are more CpGs in CTCF motifs in group 1 compared to group 4 (Additional file 1: Figure S5f, 288 versus $25 \mathrm{CpGs)}$, group 1 shows a lower level of CTCF enrichment than group 4 . This may be due to the confidence of attributing CpGs to a specific group. As shown in Additional file 1: Figure S6g, for all clusters, more than half of the CpGs have a high probability of being in the assigned group (>72\%). These data provide insight into CTCF binding and suggest an anticorrelation between high accessibility and high methylation.

The MA0139.1 CTCF motif incorporates two CpGs: $\mathrm{C} 2$ and/or C12 (Fig. 4b, top panel). According to the CTCF logo, we identified more CpGs at position $\mathrm{C} 12$ than C2 in the CTCF M-ChIP peaks (4884 versus 921 CpGs, respectively, considering only the CpGs covered by at least 5 reads in both M-ChIP and WGBS). Consistent with the findings from a recent study that analyzed CTCF binding using oligonucleotides rather than genomic DNA [19], CTCF M-ChIP detected higher levels of methylation at $\mathrm{C} 12$ compared to $\mathrm{C} 2$ (Fig. 4b, bottom panel, compare CTCF M-ChIP C2 versus C12, $p$ value = 1.02e-12). Importantly, CTCF M-ChIP is more suitable than WGBS for detecting the differences (Fig. 4b, bottom panel, compared to CTCF M-ChIP versus WGBS, $p$ value $=0.023$ ). In addition, we found that bi-methylation at both CpGs within the same read is slightly enriched compared to what is expected by random chance $(0.97 \%$ versus $0.05 \%$ ) (Additional file 1: Figure S7a, $X^{2}=1531, p$ value $<0.001$ ). CTCF signal intensity is relatively comparable at the four combinations of methylation, with a slight increase for $\mathrm{C} 2$ being methylated and $\mathrm{C} 12$ unmethylated (Additional file 1: Figure S7b); however, the biological significance of this remains to be determined. Nonetheless, sequence variation at the $\mathrm{C} 2$ and C12 positions appears to have no effect on methylation levels (Additional file 1: Figure S7c).

\section{KLF4 M-ChIP enables characterization of WT versus mutant KLF4 R462A binding}

Pioneer transcription factors need to access target genes that are inaccessible and whose enhancer and promoter sequences may be methylated. A recent study has shown that a minority of transcription factors (47 out of 1300 examined) including KLF4 can bind to methylated CpG sites [2]. A scatter plot of KLF4 M-ChIP in WT mESC shows that the majority of CpGs in KLF4 peaks display low peak intensity and low methylation (Fig. 4c). However, in contrast to CTCF, the small fraction of peaks with the highest peak intensity also display the highest methylation levels. The study mentioned above [2] revealed that distinct zinc fingers on KLF4 mediate KLF4's binding activity with methylated and unmethylated DNA. Residue arginine 458 on human KLF4 was shown to be important for binding to the methylated motif CCmCpGCC [2] (similar to the Jaspar motif MA0039.2 for mouse KLF4). In the mouse protein, the equivalent arginine residue lies at position 462 .

In order to investigate the binding of KLF4 to methylated DNA, we used $\mathrm{Klff}^{-/-}$mESC [20] that express either a WT or mutant version of KLF4 in which arginine 462 has been replaced by alanine (R462A) (Additional file 1: Figure S8a-b). We performed KLF4 MChIP in both WT and mutant expressing mESC in duplicates. Intersections between replicates were used to identify peaks specific to (i) WT or (ii) mutant versions of KLF4 and (iii) those that were common to both (Fig. 4d). Heatmaps confirm the binding specificity of the two versions of KLF4 and reveal the high reproducibility between duplicates (Additional file 1: Figure S8c).

We searched for mouse KLF4 motifs from the Jaspar database, using the FIMO tool from the MEME suite. The two motifs that were identified, MA0039.2 and MA0039.1, can be distinguished by the presence and absence of a CpG dinucleotide, respectively (Fig. 4e, top). The wild-type version of KLF4 has a strong preference for motif MA0039.2 while the mutant loses this preference. Overall, the mutant protein has reduced binding to both motifs (Fig. 4e, bottom).

Because of the low numbers of consensus KLF4 motifs in common and KLF4 mutant-specific peaks, we decided to focus our downstream analysis only on WT-specific peaks. M-ATAC experiments conducted in duplicates in both WT and Mutant KLF4 expressing cells show that KLF4 peaks present only in the WT condition are accessible, while Mutant only KLF4 peaks are found at inaccessible sites (Fig. 4f). This result together with the motif findings (Fig. 4e) suggests that the Mutant KLF4 binding alone occurs at inaccessible sites where there is no consensus KLF4 motif. Thus, this mutation abrogates binding at consensus KLF4 motifs. The functional significance of binding of Mutant KLF4 at ectopic sites remains to be investigated. WT-specific KLF4 peaks harbor similar DNA accessibility in both WT and Mutant conditions so it is not clear why the Mutant protein does not bind. To investigate, we analyzed DNA methylation at these sites using M-ATAC, M-ChIP, and public 
WGBS from WT mESC. The levels of methylation obtained from M-ATAC were also compared for cells expressing WT and Mutant KLF4 within the WT-specific KLF4 M-ChIP peaks. In the scatter plots shown in Fig. 4g and Additional file 1: Figure S8d, most of the CpGs display low levels of methylation in any condition (bottom left corner). Thus, methylation levels do not explain the absence of Mutant KLF4 binding at these sites.

\section{Discussion}

We developed a new method, "EpiMethylTag," that allows the simultaneous analysis of DNA methylation with ChIP-seq or ATAC-seq. EpiMethylTag can be used to analyze the methylation status and coincident accessibility or binding of other chromatin-bound transcription factors. Importantly our approach is a fast, low-input, low sequencing depth method that can be used for smaller cell populations than existing methods and can be adapted for rare cell populations. Specifically, our MChIP protocol significantly reduces the input for DNAbinding factors such as CTCF. The only published genome-wide ChIP-Bis-Seq for CTCF [12] used $100 \mathrm{ng}$ of immunoprecipitated DNA. Using a $\operatorname{Tn} 5$ transposase successfully allowed us to use less than $1 \mathrm{ng}$ of immunoprecipitated DNA followed by bisulfite conversion. The number of cells required to obtain $1 \mathrm{ng}$ of ChIPped DNA will vary depending on the protocol and the antibody used. ChIP-bisulfite [10] and BisChIP-seq [11] use lower cell numbers for H3K27me3. However, such histone modifications in general require less cells for ChIP than TFs such as CTCF or KLF4 because they cover a higher portion of the genome. Although it has not been tested, our protocol may also lower the number of cells required for $\mathrm{M}-\mathrm{ChIP}$ of histone modifications.

EpiMethylTag confirmed that as a general rule, DNA methylation rarely coexists with DNA accessibility or TF binding. Nonetheless, we found M-ATAC peaks of low signal intensity that overlapped with DNA methylation. These peaks were located predominantly in intragenic and intergenic regions and associated with low transcriptional output at gene promoters. This data identifies a class of promoters with high accessibility, high levels of methylation, high H3K4me1, low K3K4me3, and low H3K27ac (Fig. 3d). The biological relevance of such "poised promoters," remains to be determined.

Of note, a recent publication used the same design for the Methyl-ATAC aspect of EpiMethylTag method [21]. As with our approach, they show that mATAC-seq detects methylation patterns that agree with both WGBS and Omni-ATAC (improved normal ATAC-seq [22]). By comparing parental with DNMT1 and DNMT3B double knockout HCT116 cells, they identified ATAC peaks with increased accessibility that were bound by TFs only in the demethylated cells. However, they did not adapt their approach to analysis of methylated ChIPseq peaks as we have done. Here we used M-ChIP to characterize the binding of both CTCF and KLF4 to motifs in the context of DNA methylation.

Methylation within CTCF motifs is known to be anticorrelated with CTCF binding [3]. Our analysis revealed that M-ATAC peaks containing a CTCF motif have an enriched CTCF intensity at CpGs with intermediate levels of methylation as opposed to low and high levels of methylation. In addition, CTCF M-ChIP revealed that methylation at CpG $\mathrm{C} 2$ is lower than at $\mathrm{CpG} \mathrm{C} 12$, a finding that suggests methylation at $\mathrm{C} 2$ could have a stronger negative impact on CTCF binding than methylation at C12. Differences of this sort could not be detected by integrating CTCF ChIPseq with WGBS (Fig. 4b).

We further demonstrate that M-ChIP could be used to characterize the profiles and methylation status of common WT and mutant KLF4 R462A binding sites. Methylation levels do not explain the absence of Mutant KLF4 binding at these sites, and it appears that the mutant does not bind the consensus motif so we cannot investigate the relationship between methylation in the KLF4 motif and binding of WT versus Mutant KLF4 (Fig. 4f, g). While the biological significance of such differences remains to be investigated, our data demonstrate that EpiMethylTag can be used to provide information about the methylation status of the binding sites for WT and mutant proteins. This information could not be obtained by performing separate methylation and ChIP-seq experiments.

\section{Conclusion}

In sum, M-ATAC and CTCF M-ChIP demonstrate a complex interplay between accessible chromatin, DNA methylation, and TF binding that could not be detected by WGBS. EpiMethylTag can be used to provide information about the DNA sequence and chromatin context of TF binding at methylated sites and its significance to gene regulation and biological processes. This technique can also be adapted for single-cell analysis.

\section{Methods \\ Cell culture}

Mouse embryonic stem cells were provided by Matthias Stadtfeld. Briefly, KH2 embryonic stem cells (ESCs) [23] were cultured on irradiated feeder cells in KO-DMEM (Invitrogen) supplemented with L-glutamine, penicillin/ streptomycin, nonessential amino acids, $\beta$ mercaptoethanol, $1000 \mathrm{U} / \mathrm{mL}$ LIF, and 15\% FBS (ESC medium). To remove feeder cells from ESCs, cells were trypsin digested and pre-plated in ESC medium for 30 min. Supernatant containing ESCs was used for further experiments. 


\section{KLF4 expression}

Mouse KLF4 has been cloned into pHAGE2-tetO-MCSires-tdTomato vector (obtained from Matthias Stadfeld's lab, [24]) for the production of lentiviruses, using the following primers:

Fwd: 5' - gcggccgcATGGCTGTCAGCGACGCTCT

Rev:

\section{ggatccTTAAAAGTGCCTCTTCATGTGTAAGG}

KLF4 R462A mutation has been generated using the site-directed mutagenesis kit from Agilent \#210518. HEK 293T cells were used for the production of lentiviruses, obtained from ATCC (cat. No. CRL 3216). Lentiviral infection of KLF4 knockout mESC [20] was performed by spin-infection, and the cells were transferred to feeders and expanded with puromycin. After selection, KLF4 expression was induced with doxycycline $(1 \mu \mathrm{g} / \mathrm{ml})$ for 2 days. Finally, the cells were pre-seeded (30 min) to remove the feeders, and the ES cells were processed as described in the "Cell culture" section. KLF4 protein expression has been checked by western blot using an antibody from Santa Cruz (\#sc-20691, now discontinued) and using $\mathrm{H} 3$ as a loading control (antiH3, Abcam, ab1791).

\section{Assembly of the transposase}

Tn5 transposase was assembled with methylated adaptors as per the T-WGBS protocol [16]. Ten microliters of each adapter with incorporated methylated cytosines (Tn5mC-Apt1 and Tn5mC1.1-A1block; $100 \mu \mathrm{M}$ each; Additional file 2: Table S1) was added to $80 \mu \mathrm{l}$ of water and annealed in a thermomixer with the following program: $95^{\circ} \mathrm{C}$ for $3 \mathrm{~min}, 70^{\circ} \mathrm{C}$ for $3 \mathrm{~min}, 45$ cycles of $30 \mathrm{~s}$ with a ramp at $-1{ }^{\circ} \mathrm{C}$ per cycle to reach $26^{\circ} \mathrm{C}$. Fifty microliters of annealed adapters was incubated with $50 \mu \mathrm{l}$ of hot glycerol and $10 \mu \mathrm{l}$ of this mixture was incubated with $10 \mu \mathrm{l}$ of Ez-Tn5 transposase (from the EZ-Tn5 insertion kit) at room temperature for $30 \mathrm{~min}$ to assemble the transposome.

\section{ATAC-seq and M-ATAC}

ATAC-seq and M-ATAC were performed with 50,000 mESC as per the original ATAC-seq protocol [13]. Cells were washed in cold PBS and resuspended in $50 \mu$ of cold lysis buffer (10 mM Tris- $\mathrm{HCl}, \mathrm{pH} 7.4,10 \mathrm{mM} \mathrm{NaCl}$, $3 \mathrm{mM} \mathrm{MgCl}_{2}, 0.1 \%$ IGEPAL CA-630). The tagmentation reaction was performed in $25 \mu$ l of TD buffer (Illumina Cat \#FC-121-1030), $2.5 \mu \mathrm{l}$ transposase (either the Nextera transposase (ATAC-seq), or the transposase containing the methylated adaptors (M-ATAC, see section "Assembly of the transposase" for details), and $22.5 \mu \mathrm{l}$ of nuclease-free $\mathrm{H}_{2} \mathrm{O}$ at $37^{\circ} \mathrm{C}$ for $30 \mathrm{~min}$. Purified DNA (on column with the Qiagen Mini Elute kit) either bisulfite converted (M-ATAC, see section "Bisulfite conversion" for details) or directly amplified (ATAC-seq, see
"Amplification of ATAC-seq and ChIP-seq libraries" for details).

\section{ChIP-seq and M-ChIP}

ChIP-seq and M-ChIP were performed on $\mathrm{mESC}$ as per the original ChIPmentation protocol [14]. Five microliters of CTCF antibody (Millipore $07-729$ ) or $25 \mu \mathrm{l}$ of KLF4 antibody (R\&D AF3158) was combined to protein A (for CTCF) or G (for KLF4) magnetic beads and added to sonicated chromatin (from 200 to $700 \mathrm{bp}$, checked on agarose gel) from 10 million $\mathrm{mESC}$, for 3 to $6 \mathrm{~h}$ rotating in the cold room. Beads were washed as per the original ChIPmentation protocol [14]: twice with TF-WBI $(20 \mathrm{mM}$ Tris- $\mathrm{HCl} / \mathrm{pH} 7.4,150 \mathrm{mM} \mathrm{NaCl}, 0.1 \%$ SDS, $1 \%$ Triton $\mathrm{X}-100,2 \mathrm{mM}$ EDTA), twice with TFWBIII $(250 \mathrm{mM} \mathrm{LiCl}, 1 \%$ Triton X-100, 0.7\% DOC, and $10 \mathrm{mM}$ Tris- $\mathrm{HCl}, 1 \mathrm{mM}$ EDTA), and twice with cold Tris- $\mathrm{Cl} \mathrm{pH} 8.0$ to remove detergent, salts, and EDTA. During the second wash, the whole reaction was transferred to a new tube to decrease tagmentation of unspecific chromatin fragments sticking to the tube wall. Beads were resuspended in $25 \mu \mathrm{l}$ of the tagmentation reaction mix (10 mM Tris $\mathrm{pH} 8.0,5 \mathrm{mM} \mathrm{MgCl} 2$, and $10 \%$ $\mathrm{v} / \mathrm{v}$ dimethylformamide), and tagmentation was performed for $1 \mathrm{~min}$ at $37^{\circ} \mathrm{C}$ with either $1 \mu \mathrm{l}$ of the Nextera transposase (ChIP-seq) or the transposase containing the methylated adaptors (M-ChIP, see section "Assembly of the transposase" for details). Then, beads were washed twice with TF-WBI $(20 \mathrm{mM}$ Tris- $\mathrm{HCl} / \mathrm{pH} 7.4,150 \mathrm{mM}$ $\mathrm{NaCl}, 0.1 \%$ SDS, $1 \%$ Triton $\mathrm{X}-100$, and $2 \mathrm{mM}$ EDTA) and twice with TET $(0.2 \%$ Tween $-20,10 \mathrm{mM}$ Tris$\mathrm{HCl} / \mathrm{pH}$ 8.0, $1 \mathrm{mM}$ EDTA). During the last wash, the whole reaction was transferred to a new tube to decrease carry-over of tagmented unspecific fragments stuck to the tube wall. Chromatin was eluted and decrosslinked by $70 \mu \mathrm{l}$ of elution buffer $(0.5 \%$ SDS, $300 \mathrm{mM} \mathrm{NaCl}, 5$ mM EDTA, $10 \mathrm{mM}$ Tris- $\mathrm{HCl} \mathrm{pH} 8.0)$ containing $20 \mu \mathrm{g}$ of proteinase $\mathrm{K}$ for $2 \mathrm{~h}$ at $55^{\circ} \mathrm{C}$ and overnight incubation at $65^{\circ} \mathrm{C}$. Eluted and purified DNA was either bisulfite converted (CTCF M-ChIP, see section "Bisulfite conversion" for details) or directly amplified (CTCF ChIP-seq, see "Amplification of ATAC-seq and ChIP-seq libraries" for details).

\section{Bisulfite conversion}

Purified DNA was bisulfite converted following the TWGBS protocol [16] with the EZ DNA methylation kit (Zymo). Oligonucleotide replacement was performed by incubating $9 \mu \mathrm{l}$ of tagmented M-ATAC or M-ChIP purified DNA with $2 \mathrm{ng}$ of phage lambda DNA as carrier, $2 \mu \mathrm{l}$ of dNTP mix ( $2.5 \mathrm{mM}$ each, $10 \mathrm{mM}), 2 \mu \mathrm{l}$ of $10 \times$ Ampligase buffer, and $2 \mu \mathrm{l}$ of replacement oligo (Tn5mC-ReplO1, $10 \mu \mathrm{M}$; Additional file 2: Table S1) in a thermomixer with the following program: $50^{\circ} \mathrm{C}$ for 1 
min, $45^{\circ} \mathrm{C}$ for $10 \mathrm{~min}$, ramp at $-0.1^{\circ} \mathrm{C}$ per second to reach $37^{\circ} \mathrm{C}$. One microliter of T4 DNA polymerase and $2.5 \mu \mathrm{l}$ of Ampligase were added, and the gap repair reaction was performed at $37^{\circ} \mathrm{C}$ for $30 \mathrm{~min}$. DNA was purified using SPRI AMPure XP beads with a bead-tosample ratio of 1.8:1 and eluted in $50 \mu \mathrm{l}$ of $\mathrm{H}_{2} \mathrm{O}$. Five microliters was kept as an unconverted control sample, and $45 \mu \mathrm{l}$ was bisulfite converted using the EZ DNA methylation kit (Zymo). Briefly, the gap repair reaction was performed by adding $5 \mu \mathrm{l}$ of M-dilution buffer and 15 min incubation at $37^{\circ} \mathrm{C}$, and bisulfite treatment was performed by adding $100 \mu \mathrm{l}$ of liquid CT-conversion reagent in a thermomixer with the following program: 16 cycles of $95^{\circ} \mathrm{C}$ for $15 \mathrm{~s}$ followed by $50^{\circ} \mathrm{C}$ for $1 \mathrm{~h}$. Converted DNA was purified on a column and amplified (see section "Amplification of M-ATAC and M-ChIP libraries" for details).

\section{Amplification of ATAC-seq and ChIP-seq libraries}

Purified DNA $(20 \mu \mathrm{l})$ was combined with $2.5 \mu \mathrm{l}$ of each primer and $25 \mu \mathrm{l}$ of NEB Next PCR master mix as per the original ATAC-seq protocol [13]. For ATAC-seq, DNA was amplified for 5 cycles and a monitored quantitative PCR was performed to determine the number of extra cycles needed not exceeding 12 cycles in total to limit the percentage of duplicated reads. DNA was purified on a column with the Qiagen Mini Elute kit. For ChIP-seq, DNA was amplified as per the ChIPmentation protocol [14] in a thermomixer with the following program: $72{ }^{\circ} \mathrm{C}$ for $5 \mathrm{~min} ; 98^{\circ} \mathrm{C}$ for $30 \mathrm{~s} ; 14$ cycles of $98^{\circ} \mathrm{C}$ for $10 \mathrm{~s}, 63^{\circ} \mathrm{C}$ for $30 \mathrm{~s}$ and $72{ }^{\circ} \mathrm{C} 30 \mathrm{~s}$; and a final elongation at $72^{\circ} \mathrm{C}$ for $1 \mathrm{~min}$. DNA was purified using SPRI AMPure XP beads with a bead-to-sample ratio of 1:1 and eluted in $20 \mu \mathrm{l}$ of $\mathrm{H}_{2} \mathrm{O}$.

\section{Amplification of M-ATAC and M-ChIP libraries}

Purified converted DNA was amplified as per the original T-WGBS protocol [16]. Briefly, $10 \mu \mathrm{l}$ of DNA was combined with $1.25 \mu \mathrm{l}$ of each primer $(25 \mu \mathrm{M}$ each) and $12.5 \mu \mathrm{l}$ of high-fidelity system KAPA HiFi uracil+ PCR master mix. DNA was amplified for 5 cycles, and a monitored quantitative PCR was performed to determine the number of extra cycles needed, not exceeding 12 cycles in total to limit the percentage of duplicated reads.

\section{Sequencing of the libraries and data processing}

For ATAC-seq, ChIP-seq, M-ATAC, and M-ChIP, libraries were quantified using Kapa qPCR kit and sequenced using the HiSeq 2500 for paired-end 50-bp reads. ChIP-seq for histone modifications in $\mathrm{MESC}$ were downloaded from GEO (H3K4me1: GSM1000121, H3K27ac: GSM1000126, H3K4me3: GSM1000124). Data processing was performed as per the pipeline available on Github (https://github.com/skoklab/EpiMethylTag).
Briefly, reads were trimmed using trim-galore/0.4.4, and aligned to the mm10 assembly of mouse genome using bowtie2 [25] for ChIP-seq and ATAC-seq, and using Bismark/0.18.1 (bowtie2) [26] for M-ChIP and M-ATAC to account for bisulfite conversion. Reads with quality < 30 and duplicates were removed using Samtools/1.3 [27]. Peaks were called using Macs/2.1.0 [28] with the following parameters: --qvalue 0.01 --nomodel --shift 0 -B --call-summits. Narrow peaks were considered for further analysis. Bigwigs were generated from bam files with RPKM normalization using Deeptools [29] for visualization on IGV.

\section{Bioinformatic analysis of data}

The distribution of fragment lengths was assessed with Deeptools/2.3.3 with option "--maxFragmentLength 1000 ", and Pearson correlations of read counts with Deeptools/2.3.3 and default parameters. Heatmaps and average profiles were performed on merged bigwig files using Deeptools/2.3.3. Default parameters from Bismark/0.18.1 (Bowtie2) [26] were used to generate coverage files containing methylation information. Only cytosines in a CpG context were used for subsequent analysis. For Fig. 3d and Additional file 1: Figure S5d, e, the plots were centered on CpGs in M-ATAC peaks from the different groups highlighted in Fig. 3a. For Fig. 4a, lists of CpGs were subsampled using BEDTools [30] to consider only the CpGs inside CTCF motifs, and the average plots were centered on those CpGs. Genomic annotations were performed using ChIPseeker [31]. CTCF motif locations in CTCF M-ChIP/ChIP and M-ATAC, and KLF4 motifs in M-ChIP peaks were determined using the FIMO tool from MEME [32], with the motif PWM from Jaspar database (MA0139.1 for CTCF and MA0039.1 and MA0039.2 for KLF4). PWM was manually modified to look at methylation frequency at different combinations of $\mathrm{C} 2$ and $\mathrm{C} 12$ dinucleotides of CTCF motif. Scripts are available on Github (https:// github.com/skoklab/EpiMethylTag). In order to account for possible lack of specificity of the anti-KLF4 antibody, we filtered out ChIP-seq peaks present in $\mathrm{Klf4}^{-/}$cells. Peaks shared or specific to either WT or mutant KLF4 were identified using BEDTools [30]. For the ChIP enrichment versus $\mathrm{CpG}$ methylation plots, we plotted the peak score versus the beta values of the $\mathrm{CpG}$ probes within the peaks, using peaks called via MACS2 for CTCF (Additional file 1: Figure S6b) and via PeaKDEck for KLF4 (Fig. 4c).

To quantify the probability of clustering CpG probes into low, medium, and highly methylated groups, we assumed that beta values (i.e., the sampling mean) are normally distributed with the mean beta value $(b)$ and variance $(b(1-b)) /((n-1))$ where $n$ is the total number of reads. This allows us to quantify the probability that each 
probe belongs to its designated cluster as $P\left(b<C_{h}\right)-P(b<$ $C_{l}$ ) where $C_{h}$ and $C_{l}$ are the high and low thresholds of the cluster respectively. In Additional file 1: Figure S6g, the points and corresponding contours are colored based on their designated cluster. The $x$-axis is the beta value and the $y$-axis is the probability that beta lies within the cluster limits. For all clusters, more than half of the CpGs have a high probability of being in the assigned group (> 72\%).

\section{Supplementary information}

Supplementary information accompanies this paper at https://doi.org/10. 1186/s13059-019-1853-6.

Additional file 1: Supplementary Figure S1-S8.

Additional file 2: Supplementary Table S1-S5.

\section{Acknowledgements}

The authors thank the New York University School of Medicine High Performance Computing (HPC) for computing technical support, and Adriana Heguy and the Genome Technology Center (GTC) core for sequencing efforts.

\section{Additional information}

Peer review information: Barbara Cheifet was the primary editor on this article and managed its editorial process and peer review in collaboration with the rest of the editorial team.

\section{Authors' contributions}

$\mathrm{PL}$ and FI designed the EpiMethylTag experiments. SV, VS, and DCDG designed and performed the experiments involving KLF4. PL performed the ChIP/M-ChIP/ATAC/M-ATAC experiments. GS, MC, and K-TK developed the analytical pipeline. PL, GS, FI, TS, SB, MC, and K-TK analyzed the data. JS, EA, MS, and JS supervised the study. PL and JS wrote the paper. All authors read and approved the final manuscript.

\section{Funding}

This work was supported by 1R35GM122515 (J.S), AACR Takeda Multiple Myeloma fellowship (P.L), and the National Cancer Center (P.L).

\section{Availability of data and materials}

All raw and processed sequencing data generated in this study have been submitted to the NCBI Gene Expression Omnibus (GEO) [33].

The following datasets were downloaded from GEO: ChIP-seq in mESC: H3K4me1: GSM1000121, H3K27ac: GSM1000126, H3K4me3: GSM1000124; WGBS and CTCF ChIP-BisSeq in mESC: GSE39739.

The pipeline for data processing is available on Github (https://github.com/ skoklab/EpiMethylTag) [34].

\section{Ethics approval and consent to participate}

Not applicable.

\section{Competing interests}

The authors declare they have no competing interests.

\section{Author details}

${ }^{1}$ New York University Langone Health, New York, NY, USA. ${ }^{2}$ New York Genome Center, New York, NY, USA. ${ }^{3}$ Meyer Cancer Center, Weill Cornell Medicine, New York, NY, USA. ${ }^{4}$ Laura and Isaac Perlmutter Cancer Center, NYU School of Medicine, New York, NY, USA. ${ }^{5}$ Skirball Institute of Biomolecular Medicine, Department of Cell Biology, Helen L. and Martin S. Kimmel Center for Biology and Medicine, Laura and Isaac Perlmutter Cancer Center, New York, NY, USA. 'Sanford I. Weill Department of Medicine, Sandra and Edward Meyer Cancer Center, Weill Cornell Medicine, New York, NY, USA. ${ }^{7}$ Institute of Computational Biomedicine, Weill Cornell Medicine, New York, NY, USA
Received: 28 June 2019 Accepted: 4 October 2019

Published online: 21 November 2019

\section{References}

1. Dor $Y$, Cedar H. Principles of DNA methylation and their implications for biology and medicine. Lancet. 2018;392:777-86.

2. Hu S, Wan J, Su Y, Song Q, Zeng Y, Nguyen HN, Shin J, Cox E, Rho HS, Woodard C, et al. DNA methylation presents distinct binding sites for human transcription factors. Elife. 2013;2:e00726.

3. Maurano MT, Wang H, John S, Shafer A, Canfield T, Lee $K$, Stamatoyannopoulos JA. Role of DNA methylation in modulating transcription factor occupancy. Cell Rep. 2015;12:1184-95.

4. Zhu H, Wang G, Qian J. Transcription factors as readers and effectors of DNA methylation. Nat Rev Genet. 2016;17:551-65.

5. Fouse SD, Shen $Y$, Pellegrini M, Cole $S$, Meissner A, Van Neste $L$, Jaenisch $R$, Fan G. Promoter CpG methylation contributes to ES cell gene regulation in parallel with Oct4/Nanog, PCG complex, and histone H3 K4/K27 trimethylation. Cell Stem Cell. 2008;2:160-9.

6. Natarajan A, Yardimci GG, Sheffield NC, Crawford GE, Ohler U. Predicting cell-type-specific gene expression from regions of open chromatin. Genome Res. 2012;22:1711-22.

7. Meissner A, Gnirke A, Bell GW, Ramsahoye B, Lander ES, Jaenisch R. Reduced representation bisulfite sequencing for comparative high-resolution DNA methylation analysis. Nucleic Acids Res. 2005;33:5868-77.

8. Kelly TK, Liu Y, Lay FD, Liang G, Berman BP, Jones PA. Genome-wide mapping of nucleosome positioning and DNA methylation within individual DNA molecules. Genome Res. 2012;22:2497-506.

9. Yin Y, Morgunova E, Jolma A, Kaasinen E, Sahu B, Khund-Sayeed S, Das PK, Kivioja T, Dave K, Zhong F, et al. Impact of cytosine methylation on DNA binding specificities of human transcription factors. Science. 2017;356(6337). eaaj2239.

10. Brinkman $A B, G u H$, Bartels SJ, Zhang $Y$, Matarese F, Simmer F, Marks $H$, Bock C, Gnirke A, Meissner A, Stunnenberg HG. Sequential ChIP-bisulfite sequencing enables direct genome-scale investigation of chromatin and DNA methylation cross-talk. Genome Res. 2012;22:1128-38.

11. Statham AL, Robinson MD, Song JZ, Coolen MW, Stirzaker C, Clark SJ. Bisulfite sequencing of chromatin immunoprecipitated DNA (BisChIP-seq) directly informs methylation status of histone-modified DNA. Genome Res. 2012:22:1120-7.

12. Feldmann A, Ivanek R, Murr R, Gaidatzis D, Burger $L$, Schubeler D. Transcription factor occupancy can mediate active turnover of DNA methylation at regulatory regions. PLoS Genet. 2013;9:e1003994.

13. Buenrostro JD, Giresi PG, Zaba LC, Chang HY, Greenleaf WJ. Transposition of native chromatin for fast and sensitive epigenomic profiling of open chromatin, DNA-binding proteins and nucleosome position. Nat Methods. 2013;10:1213-8

14. Schmidl C, Rendeiro AF, Sheffield NC, Bock C. ChIPmentation: fast, robust, low-input ChIP-seq for histones and transcription factors. Nat Methods. 2015;12:963-5.

15. Adey A, Shendure J. Ultra-low-input, tagmentation-based whole-genome bisulfite sequencing. Genome Res. 2012;22:1139-43.

16. Wang Q, Gu L, Adey A, Radlwimmer B, Wang W, Hovestadt V, Bahr M, Wolf $\mathrm{S}$, Shendure J, Eils R, et al. Tagmentation-based whole-genome bisulfite sequencing. Nat Protoc. 2013:8:2022-32.

17. Stadler MB, Murr R, Burger L, Ivanek R, Lienert F, Scholer A, van Nimwegen E, Wirbelauer C, Oakeley EJ, Gaidatzis D, et al. DNA-binding factors shape the mouse methylome at distal regulatory regions. Nature. 2011;480:490-5.

18. Wang H, Maurano MT, Qu H, Varley KE, Gertz J, Pauli F, Lee K, Canfield T, Weaver M, Sandstrom R, et al. Widespread plasticity in CTCF occupancy linked to DNA methylation. Genome Res. 2012;22:1680-8.

19. Hashimoto H, Wang D, Horton JR, Zhang X, Corces VG, Cheng X. Structural basis for the versatile and methylation-dependent binding of CTCF to DNA. Mol Cell. 2017:66:711-20 e713.

20. Di Giammartino DC, Kloetgen A, Polyzos A, Liu Y, Kim D, Murphy D, Abuhashem A, Cavaliere P, Aronson B, Shah V, et al. KLF4 is involved in the organization and regulation of pluripotency-associated three-dimensional enhancer networks. Nat Cell Biol. 2019:10:1179-90.

21. Spektor R, Tippens ND, Mimoso CA, Soloway PD. methyl-ATAC-seq measures DNA methylation at accessible chromatin. Genome Res. 2019:29. 969-77. 
22. Corces MR, Trevino AE, Hamilton EG, Greenside PG, Sinnott-Armstrong NA Vesuna S, Satpathy AT, Rubin AJ, Montine KS, Wu B, et al. An improved ATAC-seq protocol reduces background and enables interrogation of frozen tissues. Nat Methods. 2017;14:959-62.

23. Beard C, Hochedlinger K, Plath K, Wutz A, Jaenisch R. Efficient method to generate single-copy transgenic mice by site-specific integration in embryonic stem cells. Genesis. 2006;44:23-8.

24. Vidal SE, Amlani B, Chen T, Tsirigos A, Stadtfeld M. Combinatorial modulation of signaling pathways reveals cell-type-specific requirements for highly efficient and synchronous iPSC reprogramming. Stem Cell Reports. 2014;3:574-84

25. Langmead B, Salzberg SL. Fast gapped-read alignment with Bowtie 2. Nat Methods. 2012;9:357-9.

26. Krueger F, Andrews SR. Bismark: a flexible aligner and methylation caller for Bisulfite-Seq applications. Bioinformatics. 2011;27:1571-2.

27. Li H, Handsaker B, Wysoker A, Fennell T, Ruan J, Homer N, Marth G, Abecasis G, Durbin R, Genome Project Data Processing S. The sequence alignment/ map format and SAMtools. Bioinformatics. 2009;25:2078-9.

28. Zhang Y, Liu T, Meyer CA, Eeckhoute J, Johnson DS, Bernstein BE, Nusbaum C, Myers RM, Brown M, Li W, Liu XS. Model-based analysis of ChIP-Seq (MACS). Genome Biol. 2008;9:R137.

29. Ramirez F, Ryan DP, Gruning B, Bhardwaj V, Kilpert F, Richter AS, Heyne S, Dundar F, Manke T. deepTools2: a next generation web server for deepsequencing data analysis. Nucleic Acids Res. 2016;44:W160-5.

30. Quinlan AR, Hall IM. BEDTools: a flexible suite of utilities for comparing genomic features. Bioinformatics. 2010;26:841-2.

31. Yu G, Wang LG, He QY. ChIPseeker: an R/Bioconductor package for ChIP peak annotation, comparison and visualization. Bioinformatics. 2015;31:2382-3.

32. Bailey TL, Boden M, Buske FA, Frith M, Grant CE, Clementi L, Ren J, Li WW, Noble WS. MEME SUITE: tools for motif discovery and searching. Nucleic Acids Res. 2009;37:W202-8.

33. Skok Lhoumaud. EpiMethylTag simultaneously detects ATAC-seq or ChIP-seq signals with DNA methylation. Dataset GSE129673 Gene Expression Omnibus. 2019. https:/www.ncbi.nlm.nih.gov/geo/query/acc.cgi?acc=GSE129673. Accessed 1 Oct 2019.

34. Skok. Lhoumaud. EpiMethylTag simultaneously detects ATAC-seq or ChIPseq signals with DNA methylation. Github 2019. https:/github.com/skoklab/ EpiMethylTag. Accessed 1 Oct 2019

\section{Publisher's Note}

Springer Nature remains neutral with regard to jurisdictional claims in published maps and institutional affiliations.

\section{Ready to submit your research? Choose BMC and benefit from}

- fast, convenient online submission

- thorough peer review by experienced researchers in your field

- rapid publication on acceptance

- support for research data, including large and complex data types

- gold Open Access which fosters wider collaboration and increased citations

- maximum visibility for your research: over $100 \mathrm{M}$ website views per year

At $\mathrm{BMC}$, research is always in progress.

Learn more biomedcentral.com/submissions 